\title{
Oops! Resolving social dilemmas in frontotemporal dementia
}

\author{
Paul J Eslinger, Peachie Moore, Vanessa Troiani, Shweta Antani, Katy Cross, Shaleigh Kwok, Murray \\ Grossman
}

See end of article for authors' affiliations

......................

Correspondence to:

Paul J Eslinger, Department

of Neurology-H037, Penn

State Hershey Medical

Center, PO Box 850,

Hershey, PA 17033-0850,

USA; peslinger@psu.edu

Received 18 May 2006

Revised 20 September 2006

Accepted

22 September 2006

Published online first

29 September 2006

\begin{abstract}
Objectives: Our social cognition model posits that social knowledge and executive resources guide interpersonal decision making. We investigated this model by examining the resolution of standardised social dilemmas in patients with a social and executive disorder (SOC/EXEC) caused by frontotemporal dementia (FTD).

Methods: Patients with SOC/EXEC $(n=12)$ and those with progressive aphasia $(A P H, n=14)$ completed measures requiring resolution of social dilemmas (Guilford's Cartoon Predictions Test), social cognition (theory of mind false belief vignettes and a behavioural rating measure of empathy) and executive measures of cognitive flexibility (Visual Verbal Test). Regression analysis related judgments of social dilemmas to cortical volume using voxel based morphometry of high resolution structural MRI.

Results: Patients with SOC/EXEC were impaired in judgments of social dilemmas as well as theory of mind, self-awareness of empathy and cognitive flexibility. Patients with APH were much less impaired in the social and cognitive measures. There were strong correlations among social dilemma, theory of mind and mental flexibility measures in patients with SOC/EXEC, and stepwise regression showed that mental flexibility was most predictive of social dilemma judgments. Social dilemma impairments in the SOC/EXEC sample correlated with cortical atrophy in the orbital frontal, superior temporal, visual association and posterior cingulate regions of the right hemisphere.

Conclusions: Deficits in patients with SOC/EXEC in resolving social dilemmas are related to depleted executive resources and social knowledge that appear to arise from disease that interrupts a right frontaltemporal neural network crucial for mediating social cognition.
\end{abstract}

$\mathrm{P}$ rogressive social impairments are prominent clinical features of frontotemporal dementia (FTD). This is most evident in the subgroup manifesting disabling personality, behavioural and executive function disorders, with disease predominantly in the right frontal and anterior temporal regions (termed social and executive disorder (SOC/EXEC)), in contrast with FTD patients with prominent language disorders and left hemisphere disease (termed APH). ${ }^{1-4}$

We evaluated the basis for impaired social cognition in FTD using the Cartoon Predictions Test ${ }^{5}$ which assesses ability to choose the most likely outcome of a pictured social dilemma. We hypothesised that patients with SOC/EXEC would show greater deficits on objective measures of social judgment than $\mathrm{APH}$ and healthy controls, and that selecting the optimal outcome of a social dilemma would be related to social knowledge and executive resources in a social executor framework. ${ }^{6}$ The knowledge component concerns social perception, reasoning and actions within the interpersonal problem, including the roles and behaviours of characters, situational variables and the consequences of chosen actions. This is intimately related to theory of mind (ToM) processing, which is impaired in patients with SOC/EXEC. ${ }^{78}$ Therefore, we administered ToM measures requiring first order and second order beliefs and interpretation. We also suspected that empathic sensitivity would influence perception and consideration of social situations and actions, and therefore we included behavioural ratings of empathy. The second domain we implicate in resolving social dilemmas is cognitive flexibility, an executive resource supporting divergent thinking, consideration of problem solving options and empathy. ${ }^{9}$ We used the Visual Verbal Test (VVT) to assess abstraction and flexibility of relational reasoning during visual problem solving. ${ }^{10}{ }^{11}$ Finally, MRI voxel based morphometry in patients with SOC/EXEC examined whether social dilemma judgments are related to a frontal-temporal network that underlies social cognition.

\section{MATERIALS AND METHODS}

\section{Subjects}

Twenty-six patients were diagnosed according to modifications of published criteria for FTD. ${ }^{12-15}$ Other dementia causes were excluded by clinical examination, and blood and imaging tests. Informed consent was provided by participants and caregivers according to a protocol approved by the University of Pennsylvania Institutional Review Board. Two independent examiners classified FTD patients into subgroups of SOC/EXEC and $\mathrm{APH}$ (including progressive non-fluent aphasia and semantic dementia) patients. All subjects with SOC/EXEC completed brain MRI for voxel based morphometry, 9 of 12 completed cognitive measures and 8 of 12 completed empathy ratings with caregivers. Patients with SOC/EXEC were marginally younger than controls $(\mathrm{p}<0.05)$ and those with SOC/EXEC and APH had lower Mini-Mental Status Examination scores $(\mathrm{p}<0.0001)$ (table 1$)$.

\section{Cartoon predictions}

A shortened 10 item version of Form $\mathrm{A}^{5}$ was developed to assess prediction of social consequences. Subjects decided the thoughts, feelings and intentions of cartoon characters in social situations (eg, a diner finding a fly in his soup) and chose the next likely event from three choices (correct next action resolving the interpersonal problem, a socially inappropriate

Abbreviations: APH, patients with frontotemporal dementia with progressive non-fluent aphasia or semantic dementia; FTD, frontotemporal dementia; SOC/EXEC, social and executive disorder; ToM, theory of mind; VVT, Visual Verbal Test 


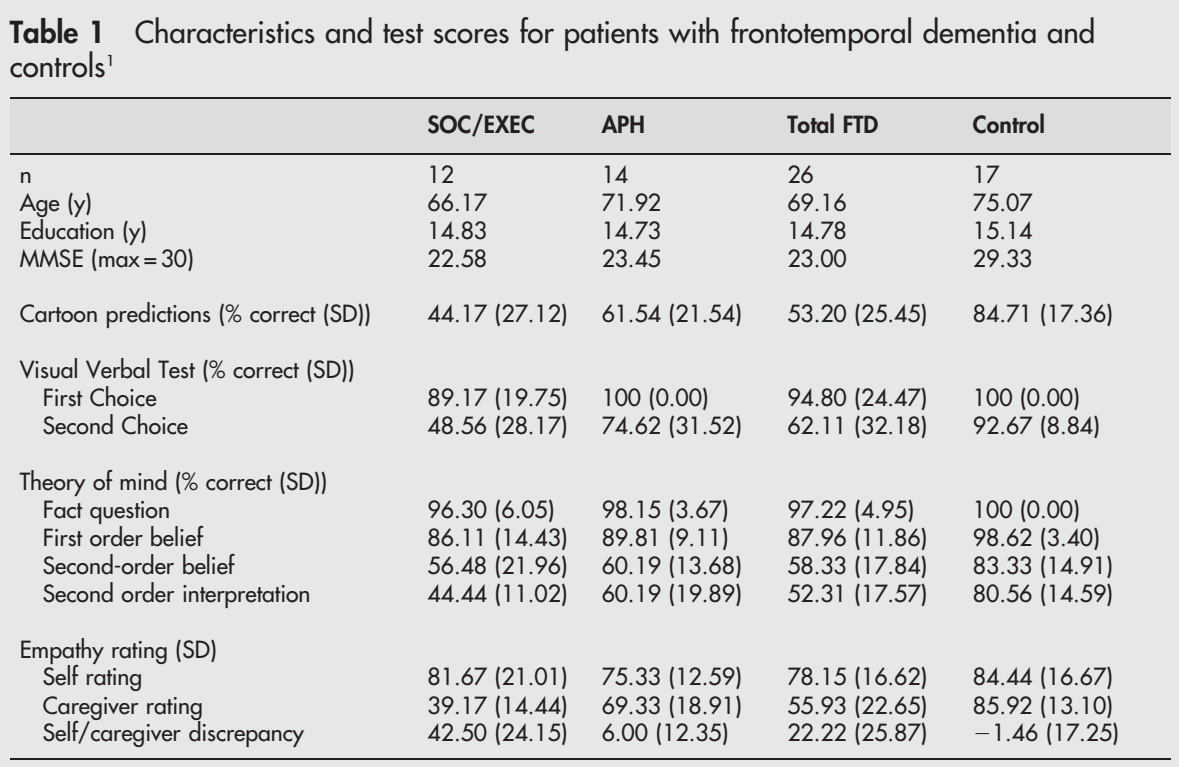

APH, frontotemporal dementia patients with progressive non-fluent aphasia or semantic dementia; FTD, frontotemporal dementia; SOC/EXEC, frontotemporal dementia patients with a disorder of social comportment and executive functioning.

Values are mean (SD).

choice and a suboptimal choice). Performance was not timed. A practice item established that participants understood the task.

\section{Theory of mind}

Twelve vignettes assessed facts and beliefs about characters in social situations. ${ }^{16}$ Each scenario was presented with lie and joke conditions that required contrasting decisions about a main character's first order beliefs, and the second order beliefs that another character held of the main character. In the lie condition, the story's agent was not aware that they were observed in the offending act; in the joke condition, the agent was aware that they were observed by the confronting character. Second order belief questions required interpretation of whether the character was lying to avoid getting caught or joking to cover up embarrassment. Vignettes were read aloud and concurrently presented in writing. A practice vignette established that participants understood the task.

\section{Visual verbal test}

This non-social measure of executive resources required abstraction and cognitive flexibility. Stimulus sets were four geometric designs on a single page. ${ }^{10}{ }^{11}$ Three designs were categorised in two different ways based on similarity in colour, shape, size or orientation. Participants identified how three designs were alike, then how three designs were alike in another way (eg, three designs black, three designs triangular). First choice accuracy ranged from 0 to 10 . Second choice accuracy was computed relative to the first choice score (range $0-100 \%$ ). Ten stimulus sets were presented. A practice item ensured comprehension.

\section{Empathy ratings}

Patients/caregivers and controls/spouses each completed three questions assessing empathic behaviours in daily social settings. ${ }^{17}$ Discrepancies were computed to measure metacognitive awareness. Ratings varied from 0 to 100 , with higher scores representing more adept empathic behaviour.

\section{Imaging procedure}

In patients with SOC/EXEC, high resolution structural MRI images were obtained using a Siemens 3.0-T MRI scanner. After rapid sagittal Tl weighted imaging to determine patient position, high resolution Tl weighted 3D MPRAGE images were acquired with a repetition time of $1620 \mathrm{~ms}$, echo time of $30 \mathrm{~ms}, 1 \mathrm{~mm}$ slice thickness, flip angle of $15^{\circ}$, matrix size of $192 \times 256$ and rectangular field of view giving an in-plane resolution of $1.0 \times 1.0 \mathrm{~mm}$. Brain volumes were registered in SPM99 ${ }^{18}$ using 12 parameter affine registration, non-linear registration using 12 non-linear iterations and $7 \times 8 \times 7$ basis functions. Brains were normalised to the Tl template of 305 averaged brain volumes with standardised brain coordinates using a high dimensional normalisation procedure. ${ }^{19}$ Brain volumes were segmented into four tissue types (gray matter, white matter, CSF and other). The segmentation algorithm in SPM99 calculates a Bayesian probability for each tissue type voxel in the volume, based on a priori MRI information. We inspected each slice of each segmented volume to ensure that no voxels from the dural sinuses or adjacent non-brain structures were misclassified as gray matter. Using SPM99, the gray matter volume was smoothed with a $12 \mathrm{~mm}$ FWHM Gaussian filter to minimise individual gyral variations.

Voxel based morphometry in SPM99 analysed brain volumes. ${ }^{20} \mathrm{~A}$ proportional analysis threshold included only voxels with $40 \%$ or more of the grand mean value. Implicit masking was used to ignore zeros, and global calculation was based on the mean voxel value. SPM99 analyses included a two sample $t$ test routine to compare the gray matter volume of patients with SOC/EXEC with 12 healthy age matched controls. Statistical threshold for atrophy studies relative to controls was set at $\mathrm{p}<0.0001$. We did not correct for multiple comparisons because of the hypothesis driven nature of the analyses. Moreover, the small size of the voxels would make Bonferroni-like statistical correction too conservative. Instead, clusters of 100 or more adjacent voxels were considered, reflecting a statistically robust effect exceeding $\mathrm{p}<0.05$ corrected for multiple comparisons in this neuroanatomical distribution. ${ }^{21}$ Regression analysis relating cartoon prediction score to gray matter atrophy, derived from contrast of cortical volume, was compared in the SOC/EXEC and control groups. Statistical threshold for this analysis was set at $\mathrm{p}<0.001$ uncorrected, requiring $>100$ adjacent voxels/cluster. 


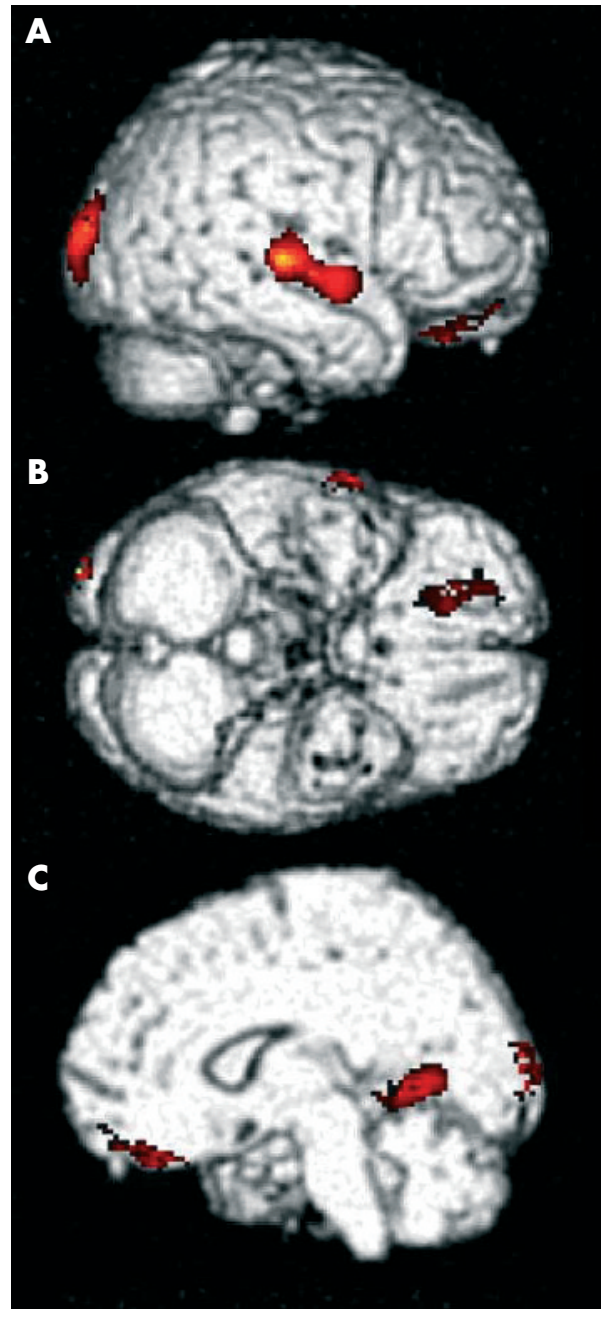

Figure 1 In patients with frontotemporal dementia with prominent social and executive impairments, regression analysis of cartoon predictions accuracy score and voxel based morphometry of cortical atrophy identified significant correlations in the right medial orbital frontal cortex (BA 11, $\mathrm{p}<0.0003$ ), right midlateral superior temporal cortex and adjacent superior temporal sulcus (BA 22, $p<0.0004$ ), right visual association cortex (BA 19, $\mathrm{p}<0.00005$ ) and right posterior cingulate (BA 30, $\mathrm{p}<0.0003$ ). (A) Right lateral; (B) ventral; (C) right medial.

\section{RESULTS}

\section{Cartoon predictions}

FTD patients scored significantly lower than controls $(\mathrm{F}=12.85, \mathrm{p}<.0001)$, with post hoc Scheffé tests showing impairments in the SOC/EXEC $(\mathrm{p}<0.0001)$ and APH $(\mathrm{p}<0.012)$ groups relative to controls (see table 1 ). Using a z score of -1.96 (equivalent to $\mathrm{p}<0.05$ ), more individual patients with $\mathrm{SOC} /$ EXEC were impaired than those with APH $\left(\chi^{2}=4.08, \mathrm{p}<0.05\right)$.

\section{Theory of mind}

FTD patients differed from controls on second order beliefs $(\mathrm{F}=4.67, \mathrm{p}=0.021)$ and second order interpretation of lies versus jokes $(\mathrm{F}=9.49, \mathrm{p}=0.001)$. Patients with SOC/EXEC performed more poorly than controls on second order beliefs $(p=0.029)$ and interpretations $(p=0.001)$, with chance response levels.

\section{Visual verbal test}

Patients with SOC/EXEC showed modest difficulty on first choice VVT accuracy, scoring below controls and patients with
APH $(\mathrm{p}<0.05)$, who did not differ. When shifting to a second categorisation, patients with SOC/EXEC were impaired relative to controls $(\mathrm{p}<0.0001)$ and those with APH $(\mathrm{p}<.037)$, who did not differ

\section{Empathy}

FTD self ratings did not differ. Caregiver ratings revealed a significant group difference $(\mathrm{F}=19.85, \mathrm{p}<0.001)$, with SOC/ EXEC profoundly lower than APH and controls $(p<.001)$, and APH marginally lower than controls $(p=0.046)$. Discrepancies differed significantly $(\mathrm{F}=5.741, \mathrm{p}=.008)$. Patients with SOC/ EXEC had greater discrepancies than those with APH or controls $(p=0.023$ and 0.002 , respectively). APH and control groups did not differ.

\section{Regression analysis in the SOC/EXEC sample}

Cartoon prediction judgments in SOC/EXEC were significantly correlated with first VVT $(\mathrm{r}=0.68, \mathrm{p}<0.05)$ and second VVT choices $(r=0.87, p<.01)$, and with ToM second order belief interpretations $(\mathrm{r}=0.72, \mathrm{p}<005)$. Second choice VVT was also significantly related to the empathy discrepancy score $(\mathrm{r}=0.71$, $\mathrm{p}<005)$. Stepwise regression analysis showed that VVT second choice was the best predictor of cartoon predictions, accounting for $74 \%$ of the variance ( $F=13.93, p=0.014)$. The combination of mean VVT score, mean ToM second order processing and empathy variables (caregiver rating and patient-caregiver discrepancy) accounted for $99 \%$ of the variance in cartoon prediction judgments of SOC/EXEC $(\mathrm{F}=554.86, \mathrm{p}=0.002)$.

\section{Neuroanatomical correlates}

Regression analysis relating cartoon prediction judgments to voxel based morphometry analysis of cortical atrophy in SOC/ EXEC revealed significant correlations in the right orbital frontal, right superior temporal, right occipital and right posterior cingulate regions (fig l).

\section{DISCUSSION}

Impaired social judgments in patients with frontotemporal dementia with a SOC/EXEC disorder were associated with ToM and cognitive flexibility deficits, and related to right hemisphere cortical atrophy in the orbital frontal, superior temporal, visual association and posterior cingulate regions. These findings support a social cognition model encompassing interacting social knowledge and executive resources in a large scale neural network involving the right frontal and temporal regions. ${ }^{6}$ Deficits in APH were less severe and infrequent, although further investigation of APH social disorders are needed..$^{22}$

Cartoon predictions monitor crucial aspects of social behaviour that are compromised in SOC/EXEC. Successful performance requires perception of characters and their mental states within specific social situations, and resolution of the social predicament by choice of subsequent actions. Patients with SOC/EXEC were profoundly impaired in social judgment and deficient in cognitive flexibility, an executive resource that was highly predictive of impaired social judgments. ToM was also highly correlated with social judgment. Together with reduced empathy, these findings suggest important inter-relationships of social and cognitive domains in a social executor framework in FTD patients with SOC/EXEC. ${ }^{6}$ Impaired social judgment in SOC/EXEC was associated with right hemisphere cortical atrophy in the orbital frontal, superior temporal gyrus/sulcus, extrastriate and posterior cingulate regions that have been linked to social and emotional impairments and to social-moral emotion, empathy, sympathy and a mentalising circuit. ${ }^{23} 24$

We hypothesise that social executors encompass knowledge and executive resources. Social knowledge elaborates the store 
of social actions, experiences and sequences that are bound by learned rules, conventions and conditional probabilities. Effective implementation of this knowledge is constrained by processing resources that are specific to the social domain (eg, ToM, empathy sensitivity) and domain-neutral executive resources (eg, cognitive flexibility). Patients with SOC/EXEC suffer progressive losses in both social knowledge and associated processing resources that cause social disability. This is related to pathology in a neural network encompassing the right orbital frontal and superior temporal regions.

\section{ACKNOWLEDGEMENTS}

This work was supported in part by the National Institutes of Health (AG17586, AG15116, and NS44266) and the Charles A Dana Foundation. Portions of this work were presented at the Conference on Frontotemporal Dementia, Philadelphia, 2004.

\section{Authors' affiliations}

P J Eslinger, Departments of Neurology, Radiology, and Neural and Behavioral Sciences, College of Medicine, Pennsylvania State University, The Milton S Hershey Medical Center, Hershey, Pennsylvania, USA P Moore, V Troiani, S Antani, K Cross, S Kwok, M Grossman, Department of Neurology, University of Pennsylvania School of Medicine, Philadelphia, Pennsylvania, USA

Competing interests: None.

\section{REFERENCES}

1 Grossman M. Frontotemporal dementia: A review. J Int Neuropsychol Soc 2002;8:564-83

2 Miller BL, Cummings JL, Villanueva-Meyer J. Frontal lobe degeneration: clinical, neuropsychological, and SPECT characteristics. Neurology 1991;41:1374-82.

3 Snowden JS, Neary D, Mann DM. Fronto-temporal lobar degeneration: frontotemporal dementia, progressive aphasia, semantic dementia. New York: Churchill Livingstone, 1996.

4 Rosen HJ, Allison SC, Schaver GF, et al. Neuroanatomical correlates of behavioural disorders in dementia. Brain 2005;128:2612-25.

5 O'Sullivan M, Guilford JP. Tests of social intelligence. Palo Alto: Consulting Psychologists Press, 1965.
6 Eslinger PJ, Grattan LM, Geder LM. Impact of frontal lobe lesions on rehabilitation and recovery from acute brain injury. NeuroRehabilitation 1995;5:161-82.

7 Gregory CA, Lough S, Stone V, et al. Theory of mind in patients with frontal variant frontotemporal dementia and Alzheimer's disease: Theoretical and practical implications. Brain 2002; 125:752-64.

8 Lough S, Kipps CM, Treise C, et al. Social reasoning, emotion, and empathy in frontotemporal dementia. Neuropsychologia 2006:44:950-8.

9 Grattan LM, Bloomer RH, Archambault FX, et al. Cognitive flexibility and empathy after frontal lobe lesion. Neuropsychiatry Neuropsychol Behav Neurol 1994;7:251-9.

10 Feldman MJ, Drawgow J. The visual verbal test manual. Beverly Hills: Western Psychological Services, 1960.

11 Stuss DT, Benson DF, Kaplan E, et al. The involvement of orbitofrontal cerebrum in cognitive tests. Neuropsychologia 1983;21:235-48.

12 The Lund and Manchester Groups. Clinical and neuropathological criteria for frontotemporal dementia. J Neurol Neurosurg Psychiatry 1994;57:416-18.

13 McKhann G, Trojanowski JQ, Grossman M, et al. Clinical and pathological diagnosis of frontotemporal dementia: Report of a work group on frontotemporal dementia and Pick's disease. Arch Neurol 2001;58:1803-9.

14 Davis KL, Price C, Moore P, et al. Evaluating the clinical diagnosis of frontotemporal degeneration: A re-examination of Neary et al, 1998. Neurology 2001;56:A144-5.

15 Neary D, Snowden JS, Gustafson L, et al. Frontotemporal lobar degeneration: A consensus on clinical diagnostic criteria. Neurology 1998;51:1546-54.

16 Winner E, Brownell HH, Happe F, et al. Distinguishing lies from jokes: Theory of mind deficits and discourse interpretation in right hemisphere-damaged patients. Brain Lang 1998:62:310-21.

17 Dywan J, Roden R, Murphy T. Orbitofrontal symptoms are predicted by mild head injury among normal adolescents. J Int Neuropsychol Soc 1995; 1:121.

18 Frackowiak RSJ, Friston KJ, Frith CD, et al. Human brain function. San Diego: Academic Press, 1997.

19 Gee JC, Ding L, Xie Z, et al. Alzheimer's disease and frontotemporal dementia exhibit distinct atrophy-behavior correlates: A computer-assisted imaging study. Acad Radiol 2003;10:1392-401.

20 Ashburner J, Friston K. Voxel-based morphometry: The methods. Neuroimage 2000;11:805-21

21 Forman SD, Cohen J, Fitzgerald M, et al. Improved assessment of significant activation in functional magnetic resonance imaging (fMRI): Use of a cluster-size threshold. Magn Reson Med 1995;33:636-47.

22 Eslinger PJ. Neurological and neuropsychological bases of empathy. Eur Neurol 1998;39:193-9.

23 Gallagher H. Reading the mind in cartoons and stories: An FMRI study of 'theory of mind' in verbal and nonverbal tasks. Neuropsychologia 2000;38:11-21.

24 Moll J, Oliveira-Souza R de, Eslinger PJ. Morals and the human brain: A working model. NeuroReport 2003;14:299-305. 